\title{
Focused parathyroidectomy for single parathyroid adenoma: a clinical account of 20 patients
}

\author{
Hany Abdelfatah El-hady ${ }^{1}$, Hisham Saleh Radwan ${ }^{2}$
}

${ }^{1}$ Lecturer, Department of Surgery, Faculty of Medicine for Girls, Al-Azhar University, Cairo, Egypt

${ }^{2}$ Professor, Department of Radiology, Faculty of Medicine, Zagazig University, Egypt and College of Medicine, Aljouf University, Sakaka, Kingdom of Saudi Arabia

Type of article: Case series

\begin{abstract}
Background: Single parathyroid adenoma is the commonest cause of primary hyperparathyroidism. Localization of the affected gland preoperatively is a critical step in management. Surgery is considered as the main line of treatment for single parathyroid adenoma. Focused technique for parathyroid excision was found by many researchers to have a good success rate even without intraoperative parathyroid hormone measurement.

Objective: The aim of this study was to assess the feasibility, safety, and adequacy of focused parathyroidectomy via an open lateral approach using preoperative positive Sestamibi (MIBI) scan and/or ultrasound without utilizing any intraoperative aiding tools.

Methods: A case-series prospective analysis of focused open parathyroidectomy for 20 patients presented to the department of surgery or referred from the orthopedic department between October 2012 and January 2015 at the Faculty of Medicine, Al-Azhar University, Cairo, Egypt, diagnosed as sporadic hyperparathyroidism with either positive MIBI scan or ultrasound were done. Normalization of the postoperative levels of serum calcium and PTH was considered as a marker of success. Data entry and analysis were done using the IBM-SPSS version 22.

Results: In this study, focused minimal access parathyroidectomy was successful in $95 \%$ with no major surgical complications, i.e., recurrent laryngeal nerve palsy or permanent hypoparathyroidism. The patient's mean age was $46.5 \pm 12$ years. The preoperative serum calcium level ranged from 8.8 to $15.2 \mathrm{mg} / \mathrm{dL}$. The parathyroid hormone level was elevated ranging from $123-2000 \mathrm{pg} / \mathrm{mL}$. In $90 \%$ of the cases, serum levels of alkaline phosphatase were elevated, while serum phosphorus was low with range $1.5-4.7 \mathrm{mg} / \mathrm{dL}$. The left inferior parathyroid gland was the most commonly involved gland (40\%). Using ultrasound and Sestamibi scan for preoperative localization was accurate in $75 \%$ and $90 \%$, respectively. When both techniques were combined localization accuracy increased to $95 \%$.

Conclusion: Our study has shown that focused parathyroidectomy is considered as a good option for single gland disease even if there are no available intraoperative helping aids, provided that good patient selection is undertaken.

Keywords: Parathyroidectomy, Focused parathyroidectomy, Single adenoma hyperparathyroidism, Parathyroid hormone
\end{abstract}

\section{Introduction}

Hyperparathyroidism due to primary cause is not an uncommon disorder. As much as $1-2 \%$ of adults could be diagnosed with primary hyperparathyroidism (PHPT). Primary hyperparathyroidism (PHPT) is the third most common endocrine disorder after diabetes and thyroid disorders with peak incidence in post-menopausal women (50-60 years) (1). Primary Hyperparathyroidism mainly targets the skeleton and kidneys. The extent of the disease is related to the timing of the diagnosis. The earlier the diagnosis, the less the extent of kidney and skeleton involvement. The skeletal affection of the disease may range from bone resorption and demineralization up to

\section{Corresponding author:}

Hany Abdelfatah El-hady, Department of Surgery, Faculty of Medicine for Girls, Al-Azhar University, Cairo, Egypt. Tel.: +966580996654, Email: hany.elhady@azhar.edu.eg and hany.elhady@yahoo.com

Received: April 14, 2018, Accepted: May 21, 2018, Published: June 2018

iThenticate screening: May 14, 2018, English editing: May 23, 2018, Quality control: May 25, 2018

This article has been reviewed / commented by three experts

(C) 2018 The Authors. This is an open access article under the terms of the Creative Commons Attribution-NonCommercialNoDerivs License, which permits use and distribution in any medium, provided the original work is properly cited, the use is non-commercial and no modifications or adaptations are made. 
pathological fracture (2). Also, PHPT Hypercalciuria is a major risk factor for developing renal stones. The patients affected by renal stones in this condition are usually younger and most of the time are males (3). Pathologically, this disorder could be an adenoma, four gland hyperplasia or very rarely parathyroid carcinoma (4). The commonest cause for PHPT is single gland adenoma ( $80-85 \%)$, however, it may be due to double adenomas (2-5\%), chief cell hyperplasia $(15-20 \%)$ or parathyroid carcinoma $(<1 \%)(5)$. Accordingly, there has been a major change in dealing with PHPT during the last two decades (4). Historically, the first parathyroidectomy was performed in 1924 by Mandl, with the exploration of 4 parathyroid glands and excision of an enlarged single parathyroid adenoma (6). Bilateral neck exploration (BNE) with visualization of all four parathyroid glands used to be the commonly applied method for successful treat of PHPT in more than $95 \%$ of cases (6). More than $80 \%$ of patients are cured after removal of a solitary parathyroid adenoma, as the well-known reason for PHPT is a single gland disease (7). The development and improvement of pre-operative parathyroid gland imaging techniques have led to further development of focused and minimally invasive surgical techniques (8). Before surgery, most centers use ultrasonography (US) and/or technetium 99m Sestamibi (MIBI) for diagnosis (9). The combined approach is more often carried out. The aim is to determine whether the patient has a single adenoma or not and therefore, select candidates for a focused approach $(9,10)$. The key for success of focused technique relies mainly on the accuracy of the preoperative localization procedures (7). The focused excision has many advantages over the traditional parathyroid exploration, namely; less pain, small skin incisions, low morbidity with less complications, and decreased hospital admission time. This has led the rapid minimally invasive/focused parathyroidectomy (MI/FP), to become the procedure of choice (11). As the half-life of PTH is roughly 5 minutes, the intraoperative monitoring of the drop of parathyroid hormone (PTH) level during resection is used as a marker of cure (12). For that, several cutoff criteria and mathematical models have been created over time. As an example, Irvin criterion which considers a PTH level drop $\geq 50 \%$ from the highest value of either pre-incision or pre-excision level at ten minutes after removal of the active gland, correctly predicted post-operative success in $96-98 \%$ of patients $(7,13)$. However, rapid PTH assay imposes additional costs and requires trained staff and equipped laboratories. Moreover, PTH baseline reference concentration is affected by the individual variability of PTH half-life, surgical manipulations and some physiologic changes during surgery as volume and type of intra-operative fluids infusions. Accordingly, some researchers reported error rates of up to $16 \%$ due to false-negative and false-positive results (12). The aim of this study was to assess the feasibility, safety, and adequacy of focused parathyroidectomy via an open lateral approach using preoperative MIBI scan and/or ultrasound without utilizing any intraoperative aiding tools to help establishing it as a simplified less invasive method for surgeons.

\section{Material and Methods}

This study was carried out on a series of 20 patients presented to the department of surgery or referred from the orthopedic department at the Faculty of Medicine, Al-Azhar University, Cairo, Egypt, between October 2012 and January 2015. Written informed consent was obtained from all participants in the study after full explanation of the benefits and risks of the procedure. They were anonymously enrolled and an ethical approval for the study was secured from the Faculty of Medicine Bioethical Committee. All included patients were diagnosed as single gland primary hyperparathyroidism. Patients' full history including age, family history, history of endocrine tumor and possible associated complications (kidney stones, and pathological fracture) were obtained from all cases. General examination and local neck examination (to detect masses or any thyroid disease) were done. Patients with failed preoperative localization, diagnosed with multiple parathyroid adenomas, associated thyroid disease that warranted a transverse cervicotomy or had previous thyroid or neck surgery, were excluded from the study. Laboratory assessment included serum calcium (total and ionized), phosphorus, parathyroid hormone level and alkaline phosphatase. Serum calcium concentration was corrected for serum albumin, using the equation, corrected $\mathrm{Ca}^{2+}=$ $[0.8 \times$ (normal albumin - patient's albumin) $]+$ serum $\mathrm{Ca}^{2+}$ level (The normal albumin level defaulted to $4 \mathrm{mg} / \mathrm{dL}$ ). PHPT was defined biochemically as the presence of hypercalcemia, hypophosphatemia and inappropriately high, normal, or elevated plasma intact PTH levels. Preoperative parathyroid adenoma image localization is performed by high-resolution neck US and MIBI scan. With the neck in extension, using high-resolution neck US (Philips IU22) with a 12-MHz linear transducer, the neck was examined from the angle of the mandible to the superior part of the anterior mediastinum. On gray scale imaging, the parathyroid adenoma was identified as a hypoechoic nodule, well distinguished from the thyroid gland. Technetium-99m Sestamibi scanning involved two-phase scanning. Detection of focal radioactivity on the 2-hour delayed image indicated a positive test. We did not conduct Single Photon Emission Computerized Tomography (SPECT) as a routine assessment (Figure 1). In cases where the findings were suggestive of single-gland disease, surgery via a focused open lateral-approach was done. In cases where the abnormal gland could not be located in the first surgical side, conversion to BNE was used. Preoperative and postoperative vocal cords assessment was done routinely. 


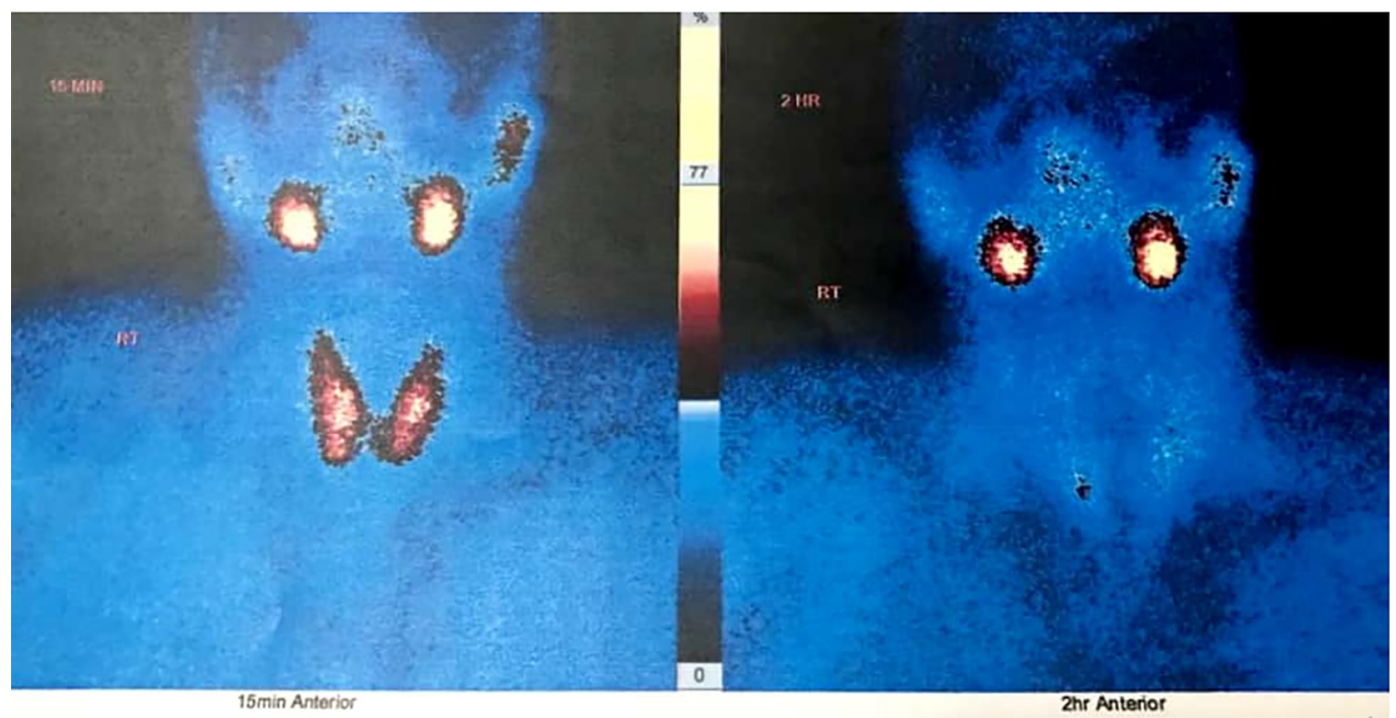

Figure 1. Fifteen minutes and 2 hours after Technetium-99m Sestamibi injection, showing delayed activity of the right (RT) inferior parathyroid gland.

Under general anesthesia with a pillow under the shoulders, depending on the localization, the incision was located. A curvilinear collar-type incision is placed transversally along the Langer's line of the skin. A small mark $1 \mathrm{~cm}$ superior to the neck crease was placed in case of superior parathyroid adenoma. Such incision was placed $1 \mathrm{~cm}$ inferior to the neck crease line in case of inferior adenoma (Figure 2). Incision through the skin and platysma was followed by creating a subplatysmal space. The sternomastoid medial margin was defined and was retracted laterally. The strap muscles were retracted medially while locating and dividing the middle thyroid vein. The space between the posterior aspect of the gland and prevertebral fascia was dissected carefully as it exposes most of the parathyroid bearing area of that side of the neck.

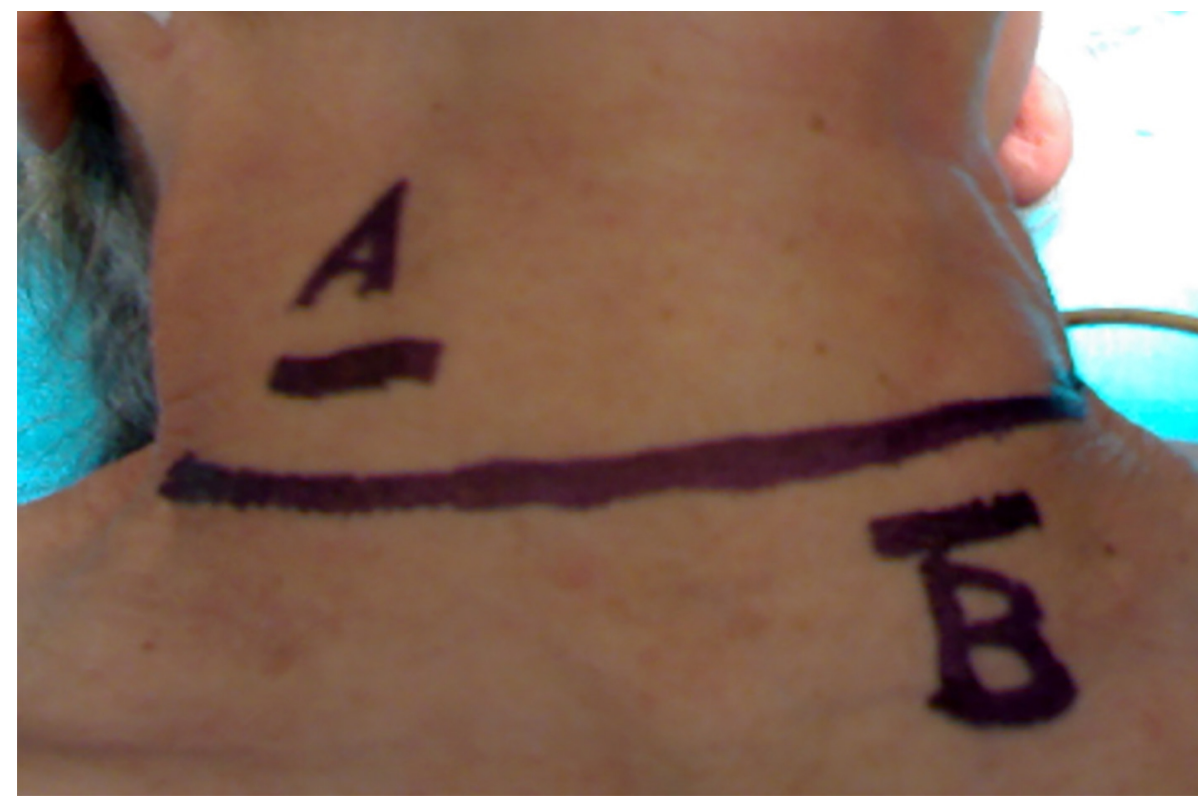

Figure 2. A curvilinear line at the site of a standard collar incision is marked. For a superior parathyroid adenoma, a small incision $1 \mathrm{~cm}$ superior to the neck crease line (A). For an inferior parathyroid adenoma, the incision is placed

$1 \mathrm{~cm}$ inferior to the neck crease line and medial to the medial margin of the sternomastoid muscle (B).

The thyroid lobe was retracted medially bringing the inferior pole of the thyroid and thymic limb in view to explore the inferior parathyroid. Careful identification and dissection of the adenoma preserving the related RLN was done 
(Figure 3). For superior parathyroid adenoma, the thyroid lobe was elevated superomedially and the superior parathyroid adenoma was located. The identified adenoma was removed intact (Figure 3) without breaching the parathyroid capsule and avoiding injury to the RLN. After resection, the removed parathyroid was histopathologically examined. Fasting calcium and PTH levels were measured on day one, sixth week and sixth month. Normalization of both values on day one was considered a full success for surgery. Persistent hypercalcemia was defined as recurrent presence of high calcium within 6 months of operation. The patients were discharged once their calcium level was stable or normal. Preoperative imaging results, postoperative complications, conversion rate, number of identified abnormal glands, operation time, length of hospital stay, and persistence and recurrence of hypercalcemia were noted. Descriptive statistics for enrolled participants are presented as counts and percentages for categorical variables, and means and standard deviations for quantitative variables. Data entry and analysis were done using IBM $\odot$ SPSS $\odot$ Statistics version 22 (IBM@ Corp., Armonk, NY, USA).

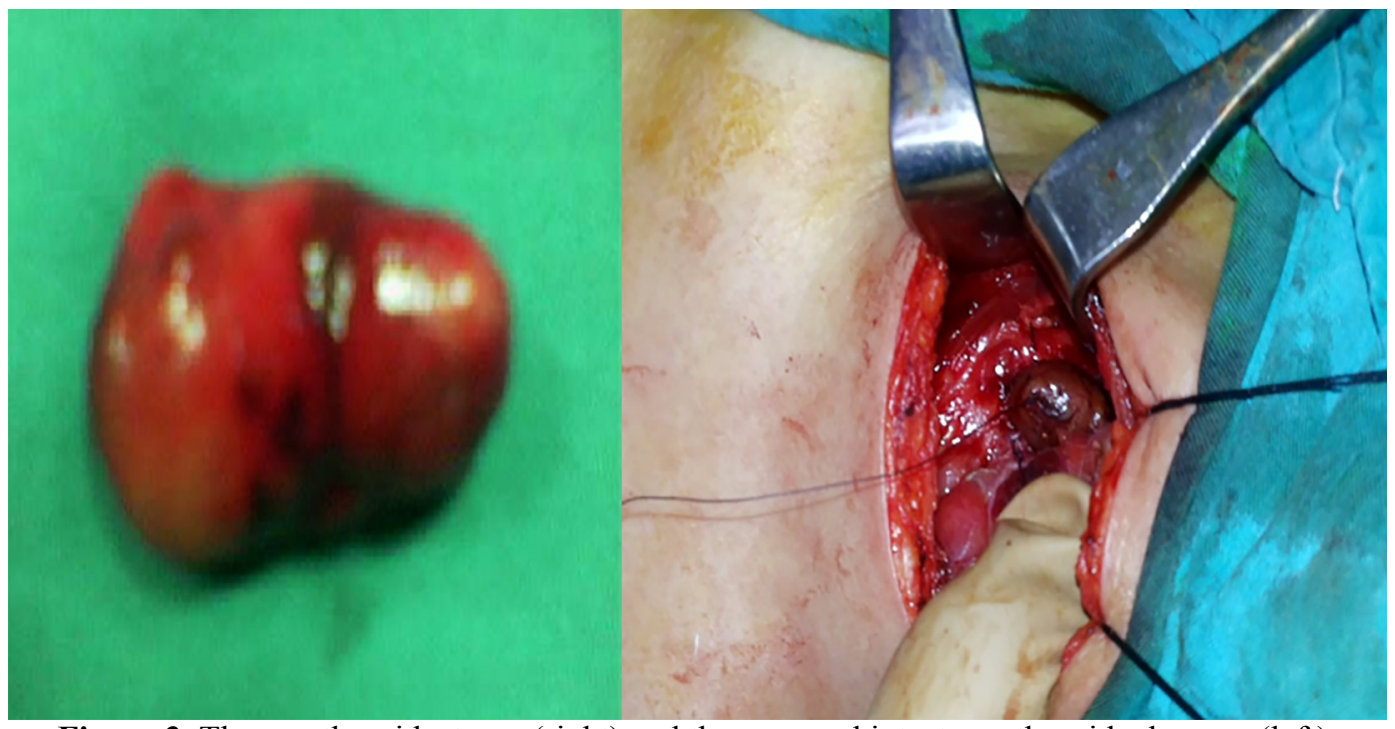

Figure 3. The parathyroidectomy (right) and the removed intact parathyroid adenoma (left).

\section{Results}

In this study, age ranged from 30 to 70 years with mean age $46.5 \pm 12$ years. Fifteen patients were females (75\%) and five $(25 \%)$ were males. Four females and one male patient were referred from the orthopedic department as their main presentation was pathological fracture $(25 \%)$ and they were diagnosed as PHPT. Other symptoms of the disease included generalized bone pain in eight cases (40\%), back pain in 5 cases $(25 \%)$ and loin pain in 2 cases $(10 \%)$. The adenoma affected the left inferior gland in eight cases (40\%), right inferior gland in 6 cases (30\%), and 3 cases $(15 \%)$ each of right superior gland and the left superior. No palpable neck swelling could be found on clinical examination. The preoperative serum calcium level ranged from 8.8 to $15.2 \mathrm{mg} / \mathrm{dL}$ (mean $11.5 \pm 2.19 \mathrm{mg} / \mathrm{dL}$ ). The parathyroid hormone level was elevated ranging from $123-2000 \mathrm{pg} / \mathrm{mL}$ (mean $473 \pm 73 \mathrm{pg} / \mathrm{mL}$ ). The serum level of alkaline phosphatase was elevated in 18 patients $(90 \%)$ with the mean of $400 \pm 93 \mathrm{U} / \mathrm{L}$ (range was 73-665 U/L). While serum phosphorus was low with mean $2.5 \pm 1.03 \mathrm{mg} / \mathrm{dL}$ (range was $1.5-4.7 \mathrm{mg} / \mathrm{dL}$ ). In nineteen out of the twenty patients $(95 \%)$ - depending on the preoperative 99m-Tc-Sestamibi scintigraphy and neck US, focused minimal access parathyroidectomy was successfully done, and failed in one case (5\%). Failed focused parathyroidectomy was attributed to the presence of adenoma in another gland rather than the operated one. This patient was re-operated by BNE with removal of the involved gland.

The operative finding in correlation to the preoperative localization by US was accurate in 15 cases (75\%) while Sestamibi scan was accurate in localization in 18 cases $(90 \%)$. When both techniques were combined, 19 cases were accurately localized (95\%). The operative time ranged from 35-60 minutes (mean 43.65 \pm 11 minutes) and hospital stay ranged from 1-3 days (mean 2.4 \pm 0.4 days). The postoperative serum levels of total serum calcium ranged from $8-11.2 \mathrm{mg} / \mathrm{dL}$ (mean $9.35 \pm 0.91 \mathrm{mg} / \mathrm{dL}$ ), phosphate levels ranged from $1.5-3.6 \mathrm{mg} / \mathrm{dL}$ (mean $2.94 \pm 0.75 \mathrm{mg} / \mathrm{dL}$ ) and PTH levels ranged from $7-128 \mathrm{pg} / \mathrm{mL}$ (mean $28 \pm 18.8 \mathrm{pg} / \mathrm{mL}$ ). There were no major surgical complications as RLN palsy or permanent hypoparathyroidism were observed. Postoperatively, there was a gradual decrease in the serum calcium levels and development of tetany in $90 \%$ of cases. Persistence of the disease occurred in 1 case (5\%) due to 
missed adenoma and this patient was re-operated by BNE technique. Seroma occurred in one case (5\%) and wound infection was observed in one case (5\%).

\section{Discussion}

Accurate localization of the parathyroid gland preoperatively is the key for successful focal parathyroid exploration. When a solitary adenoma is localized pre-operatively, minimal invasive parathyroidectomy replaces BNE as the procedure of choice (13). In our study, focused parathyroidectomy succeeded in $95 \%$ of cases without major postoperative complications. The operative time and the hospital stay were 35-60 $\mathrm{min}$ and 1 to 3 days respectively, which is less than the traditional neck exploration. As shown in a study conducted by (14), BNE versus minimally invasive procedures showed complication rate of $3.0 \%$ and $1.2 \%$, respectively, with approximately a $50 \%$ reduction in operating time and a seven-fold reduction in length of hospital stay. In this study, patients' age was found to be younger (mean age $46.5 \pm 12$ years) when compared to other studies $(4,6,10,14)$. The exact explanation of younger age at diagnosis is not clear but the non-representative small patient number included in this study or vitamin $\mathrm{D}$ deficiency, may be proposed as etiological factors. Further studies may be needed to find out the possible reasons. Nowadays, particularly in developed countries, hyperparathyroidism is diagnosed in asymptomatic cases due to routine biochemical screening (1). All the cases included in this study were complaining of disease symptoms some of them were even presented with complications. We need to increase the disease awareness and adopt biochemical screening to diagnose the cases before presenting with severe symptoms. The inferior parathyroids were more involved than the upper glands (70\%). Out of these, about $40 \%$ were located on the left side. Similar results were demonstrated by Al-Lami et al. (15) as $72.3 \%$ of his study patients were having inferior adenomas. Other studies noticed a little higher rate of inferior glands involvement $(86.7 \%)(6,16)$. In concordance to other studies, MIBI and US achieved high accuracy in locating parathyroid adenomas (17). These results encouraged the unilateral approach and minimally invasive parathyroidectomy to become a popular practice, especially with the fact that $85 \%$ of patients with primary hyperparathyroidism show uniglandular disease. MIP not only avoids more dissection, more pain, and prolonged hospital stays, it offers lower costs with comparable success rates when compared to the results of BNE.

IOPTH assay is not used in this study and it is believed by many surgeons that intra-operative assay of the level of parathyroid hormone is mandatory to ascertain complete removal of all hyperactive parathyroid glands. However, because of the longer operative time, higher cost, and a non-negligible rate of false-positive and false-negative results (especially for patients with multiglandular pathology), the added value of intra-operative parathyroid hormone monitoring has been questioned in some studies (18-22). It is evident that intraoperative PTH measurement works best in cases of single adenoma, which is usually easily localized and removed successfully by preoperative scanning and MIP without IOPTH assay, but it fails, when most needed, in the presence of multiple-gland disease. Utilizing intra-operative parathyroid hormone monitoring, improved the cure of primary hyperparathyroidism in probably $1 \%$ (22). This means that the test is overused in $99 \%$ of cases. The limitations of this study were the small number of patients and the short follow up period. More studies on a larger number of cases with longer time for follow up to assess the long-term results are planned for.

\section{Conclusions}

We suggest that focused parathyroidectomy is feasible, safe and effective as a treatment option depending only on the preoperative localization without any intraoperative aids. This technique offers good results without the disadvantages of the conventional methods. Further research is needed to assess the long term results and to find out why this disease is common in younger age groups especially in developing countries.

\section{Acknowledgments:}

The author is thankful to the Al-Azhar University Hospital (Egypt) for supporting this study. We gratefully thank Sayed M. Abdel Wahab, Professor of surgery, for his help.

\section{Conflict of Interest:}

There is no conflict of interest to be declared.

Authors' contributions:

Both authors contributed to this project and article equally. Both authors read and approved the final manuscript. 


\section{References:}

1) Pyram R, Mahajan G, Gliwa A. Primary hyperparathyroidism: skeletal and non-skeletal effects, diagnosis and management. Maturitas. 2011; 70(3): 246-55. doi: 10.1016/j.maturitas.2011.07.021. PMID: 21943558.

2) Miller PD, Bilezikian JP. Bone densitometry in asymptomatic primary hyperparathyroidism. J Bone Miner Res. 2002; 17 Suppl 2: N98-102. PMID: 12412785.

3) Khan A, Hanley D, Rizzoli R, Bollerslev J, Young J, Rejnmark L, et al. Primary hyperparathyroidism: review and recommendations on evaluation, diagnosis, and management. A Canadian and international consensus. Osteoporos Int. 2017; 28(1): 1-19. doi: 10.1007/s00198-016-3716-2. PMID: 27613721, PMCID: PMC5206263.

4) Hocevar M, Lezaic L, Rep S, Zaletel K, Kocjan T, Sever MJ, et al. Focused parathyroidectomy without intraoperative parathormone testing is safe after pre-operative localization with 18 F-Fluorocholine PET/CT. Eur J Surg Oncol. 2017; 43(1): 133-7. doi: 10.1016/j.ejso.2016.09.016. PMID: 27776943.

5) Seeliger B, Alesina PF, Koch JA, Hinrichs J, Meier B, Walz MK. Diagnostic value and clinical impact of complementary CT scan prior to surgery for non-localized primary hyperparathyroidism. Langenbecks Arch Surg. 2015; 400(3): 307-12. doi: 10.1007/s00423-015-1282-2. PMID: 25702138.

6) Usta A, Alhan E, Cinel A, Türkyılmaz S, Erem C. A 20-year study on 190 patients with primary hyperparathyroidism in a developing country: Turkey experience. Int Surg. 2015; 100(4): 648-55. doi: 10.9738/INTSURG-D-14-00094.1. PMID: 25875546, PMCID: PMC4400933.

7) Calò PG, Medas F, Loi G, Erdas E, Pisano G, Nicolosi A. Feasibility of unilateral parathyroidectomy in patients with primary hyperparathyroidism and negative or discordant localization studies. Updates Surg. 2016; 68(2): 155-61. doi: 10.1007/s13304-015-0342-z. PMID: 26826082.

8) Karakas E, Kann S, Höffken H, Bartsch DK, Celik I, Görg C, et al. Does contrast-enhanced cervical ultrasonography improve preoperative localization results in patients with sporadic primary hyperparathyroidism? J Clin Imaging Sci. 2012; 2: 64. doi: 10.4103/2156-7514.103054. PMID: 23230546, PMCID: PMC3515932.

9) Guerin C, Lowery A, Gabriel S, Castinetti F, Philippon M, Vaillant-Lombard J, et al. Preoperative imaging for focused parathyroidectomy: making a good strategy even better. Eur J Endocrinol. 2015; 172(5): 51926. doi: 10.1530/EJE-14-0964. PMID: 25637075.

10) Kandil E, Malazai AJ, Alrasheedi S, Tufano RP. Minimally invasive/focused parathyroidectomy in patients with negative sestamibi scan results. Arch Otolaryngol Head Neck Surg. 2012; 138(3): 223-5. doi: 10.1001/archoto.2011.1419. PMID: 22351855.

11) Kandil E, Malazai AJ, Alrasheedi S, Tufano RP. Minimally invasive/focused parathyroidectomy in patients with negative sestamibi scan results. Arch Otolaryngol Head Neck Surg. 2012; 138(3): 223-5. doi: 10.1001/archoto.2011.1419. PMID: 22351855.

12) Calò PG, Pisano G, Loi G, Medas F, Barca L, Atzeni M, et al. Intraoperative parathyroid hormone assay during focused parathyroidectomy: the importance of 20 minutes measurement. BMC Surg. 2013; 13(1): 36. doi: 10.1186/1471-2482-13-36. PMID: 24044556, PMCID: PMC3848580.

13) Minisola S, Cipriani C, Diacinti D, Tartaglia F, Scillitani A, Pepe J, et al. Imaging of the parathyroid glands in primary hyperparathyroidism. Eur J Endocrinol. 2016; 174(1): D1-8. doi: 10.1530/EJE-15-0565. PMID: 26340967.

14) Udelsman R. Six hundred fifty-six consecutive explorations for primary hyperparathyroidism. Ann Surg. 2002; 235(5): 665. doi: 10.1097/00000658-200205000-00008. PMID: 11981212, PMCID: PMC1422492.

15) Al-Lami A, Riffat F, Alamgir F, Dwivedi R, Berman L, Fish B, et al. Utility of an intraoperative ultrasound in lateral approach mini-parathyroidectomy with discordant pre-operative imaging. Eur Arch Otorhinolaryngol. 2013; 270(6): 1903-8. doi: 10.1007/s00405-012-2284-0. PMID: 23183852.

16) Afzal A, Gauhar TM, Butt WT, Khawaja AA, Azim KM. Management of hyperparathyroidism: a five year surgical experience. J Pak Med Assoc. 2011; 61(12): 1194-8. PMID: 22355965.

17) Haber RS, Kim CK, Inabnet WB. Ultrasonography for preoperative localization of enlarged parathyroid glands in primary hyperparathyroidism: comparison with 99 mtechnetium sestamibi scintigraphy. Clin Endocrinol (Oxf). 2002; 57(2): 241-9. doi: 10.1046/j.1365-2265.2002.01583.x.

18) Wong W, Foo FJ, Lau MI, Sarin A, Kiruparan P. Simplified minimally invasive parathyroidectomy: a series of 100 cases and review of the literature. Ann R Coll Surg Engl. 2011; 93(4): 290-3. doi: 10.1308/003588411X571836. PMID: 21944794, PMCID: PMC3363078.

19) Badii B, Staderini F, Foppa C, Tofani L, Skalamera I, Fiorenza G, et al. Cost-benefit analysis of the intraoperative parathyroid hormone assay in primary hyperparathyroidism. Head Neck. 2017; 39(2): 241-6. doi: 10.1002/hed.24567. PMID: 27557453. 
20) Suliburk JW, Sywak MS, Sidhu SB, Delbridge LW. 1000 minimally invasive parathyroidectomies without intra - operative parathyroid hormone measurement: lessons learned. ANZ J Surg. 2011; 81(5): 362-5. doi: 10.1111/j.1445-2197.2010.05488.x. PMID: 21518187.

21) Chow TL, Choi CY, Lam SH. Focused parathyroidectomy without intra-operative parathyroid hormone monitoring for primary hyperparathyroidism: results in a low-volume hospital. J Laryngol Otol. 2015; 129(8): 788-94. doi: 10.1017/S0022215115000651. PMID: 26072937.

22) Pang $T$, Stalberg $P$, Sidhu S, Sywak M, Wilkinson M, Reeve $T$, et al. Minimally invasive parathyroidectomy using the lateral focused mini - incision technique without intraoperative parathyroid hormone monitoring. Br J Surg. 2007; 94(3): 315-9. doi: 10.1002/bjs.5608. PMID: 17205496. 